\title{
SULLA SCOPERTA DI UN'ALTRA CHIESA PALEOCRISTIANA NELL'ISOLA DI KRK (VEGLIA)
}

\section{RANKO STARAC}

UDC 904(497.5):726.54

R. Starac

Preliminary communication

F. Kurelca 6

Manuscript received: 15. 04. 1996.

Revised manuscript accepted: 22. 04. 1996.

Rijeka

Croatia

Nel suo intervento l'autore riporta i risultati del sondaggio sperimentale della basilica paleocristiana di Sv, Nikola, recentemente scoperta nella località di "Mire", fra Jurandvor e Baška (isola di Krk/Veglia).

Il territorio della vallata di Baška è ricco di monumenti dei periodi antico, tardo antico e paleobizantino ${ }^{1}$. Oltre alle abitazioni di peregrini attestate dall'epoca preistorica, quali Sv. Ivan (S. Giovanni), Bag, Bunculuka o Grad (Borgo) presso Batomlje, nel corso dell'antichità vengono formati, lungo la campagna fertile, nuovi abitati nelle zone di "Skopalj" e "Mire", come anche un piccolo complesso rurale nel luogo dove più tardi sorgerà l'abbazia benedettina di Sv. Lucija (S. Lucia) a Jurandvor.

Durante le recenti ricerche archeologiche dei resti dell'abbazia benedettina di Sv. Lucija (S.Lucia) è stato sottoposto a sistematica ricognizione un vasto territorio attorno all'abitato di Jurandvor. Oltre alle già note località, quali i ruderi della chiesa di Sv. Marija (S. Maria) sul Goričica² (in aperta campagna ai confini di Jurandvor) e la cappella diroccata di Sv. Križ (S.Croce) (intorno alla quale si trova una necropoli medioevale), è stata delimitata l'area di un ampio complesso del periodo antico e tardo antico in territorio di "Mire", ubicato all'estremo sud di Jurandvor, che si sviluppa lungo i due lati della strada per Baška. A un centinaio di metri dalla periferia sudorientale dell'abitato di "Mire" sono stati scoperti, nel 1970, in occasione dell'impianto di un vigneto, resti di muri. Nel

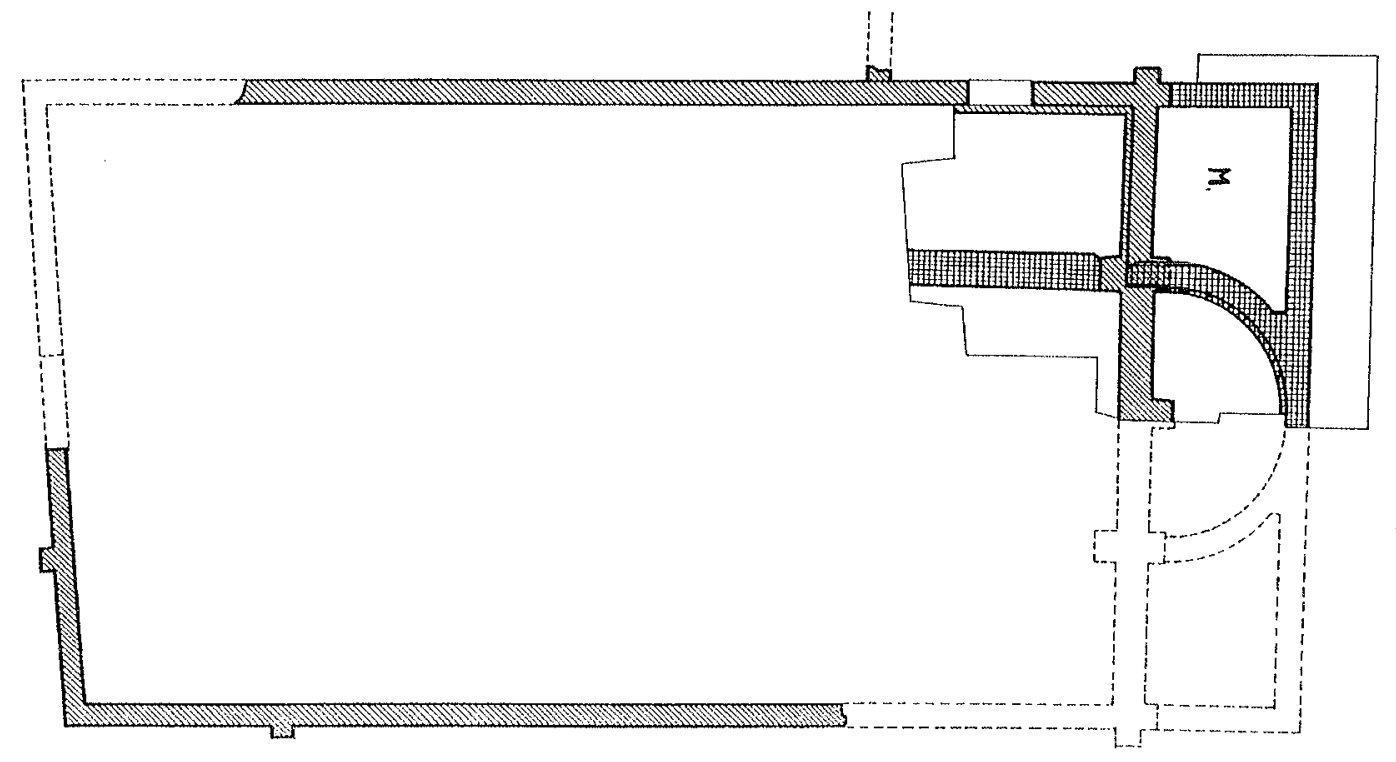

Fig. 1. Pianta della chiesa di Sv. Nikola

- le linee oblique contraddistinguono la prima fase edilizia

- il retino contraddistingue la seconda fase edilizia (V sec.)

$M=$ posizione del mosaico 


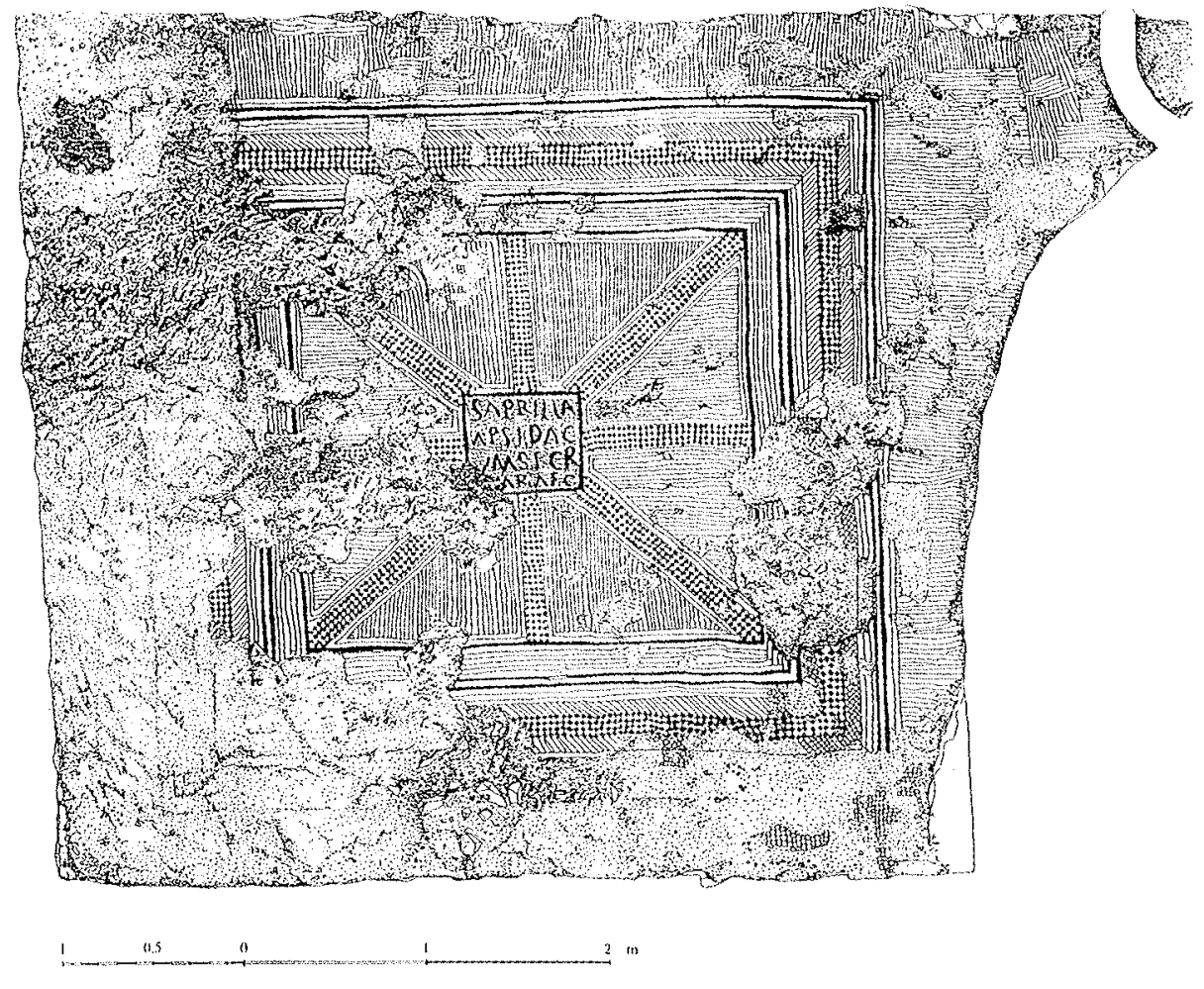

Fig. 2. Schizzo della pavimentazione a mosaico del vano sussidiario

corso di un breve sondaggio, effettuato da V. Dautova-Ruševljan ${ }^{3}$, sono stati esplorati i resti di due tombe tardoantiche in muratura, tra loro parallele, con costruzione a volta. In quella occasione la Dautova-Ruševlijan ha notato "... una costruzione dalle pareti di $70 \mathrm{~cm}$. di spessore, a forma rettangolare...", sulla cui funzione l'archeologa non ha potuto esprimersi.

Nel visitare la località sono stati notati i resti di un ampio edificio rettangolare, con pareti conservate, alte dai 50 ai 180 $\mathrm{cm}$. e spesse $55-60 \mathrm{~cm}$. Il sistema di costruzione è in contrasto con l'opus dell'antichità classica, essendo le pareti in pietra calcarea, solo in parte lavorata, legata con abbondante malta chiara. Nelle parti dei tratti conservati si scorgono lesene rettangolari, ed è visibile anche la posizione di due porte. Un ingresso è ubicato nell'asse della parete frontale (visibile lo stipite destro), mentre l'altro (largo $130 \mathrm{~cm}$.) conduce, attraverso la parete nordoccidentale laterale dell'edificio, in aperta campagna (oggi un vigneto), dove nel 1970 sono state rinvenute delle tombe. L'edificio è orientato secondo la centuriazione dell'agro di Baška risalente alla prima antichità; l'agro comprende anche dei complessi rurali, per cui l'orientamento viene ereditato anche dalle costruzioni della tarda antichità e dell'epoca paleocristiana quali la struttura di Sv. Lucija o quello della basilica sottostante la cappella di Sv. Marko (S.Marco). Il più vicino toponimo registrato presso la popolazione è quello di "Antonove kuće" o "Tonovo selo", ma la denominazione si riferisce a un cumulo di pietre ad una decina di metri più a sud, in cui si trovano frammenti di malta, di tegole e di ceramiche risalenti all'antichità. È interessante che la popolazione ignori il più antico toponimo registrato nel Catasto, che è quello di "S.Nicolo (Sv. Nikola)"; e sebbene noi per ora riprenderemo tale denominazione, bisogna comunque mantenere delle riserve sulla sua autenticità. Sulla superficie del terreno all'interno della località di "Mire" ci si imbatte in enormi quantità di pietre con resti di malta, tegole antiche, ceramiche tardoantiche e paleobizantine, come anche in frammenti di pavimentazione marmorea. $\dot{\mathrm{E}}$ stato rinvenuto anche un frammento di transenna in marmo che corrisponde ad un altro, rinvenuto in precedenza all'interno del parterre della chiesa di Sv. Lucija. Ad alcune centinaia di metri a sud della località di Sv. Nikola (dal poggiolo con la chiesetta di Sv. Mihovil (S.Michele fino alla spiaggia) sorge un altro abitato antico con necropoli, chiamato "Skopalj". Ne fa parte la basilica sottostante Sv. Marko.

Nel corso del tardo autunno del 1995 è stato effettuato un sondaggio sperimentale della parte settentrionale dell'edificio ${ }^{4}$ Esplorata una superficie di ca. $75 \mathrm{mq}$., sono stati rinvenuti resti della parte posteriore con l'apside di una chiesa paleocristiana. In base alle caratteristiche delle pareti ed alla profondità del basamento, è possibile distinguere chiaramente due fasi edilizie. Nella prima fase la costruzione presenta, all'esterno dei muri, sporgenze rettangolari a contrafforte; è edificata in breccia di pietra calcarea, il cui legante è costituito da solida malta chiara con amalgama di tegole finemente sminuzzate. L'edificio nella parte posteriore è stato successivamente prolungato e, nello spazio intermedio ottenuto, è stata ubicata l'abside semicircolare. Da ambo i lati dell'abside in questo modo vengono formati due ambienti a forma di parallelogramma irregolare. Questo corpo aggiunto ha un basamento molto meno profondo; è costruito in arenaria grigio-marrone sminuzzata a strati. La facciata dell'edificio riporta linee decorative di intonaco giallastro, caratteristiche del periodo tardoantico.

Lo spazio fra l'arco absidale, la parete posteriore della prima fase dell'edificio (attraverso la quale, nella seconda fase 


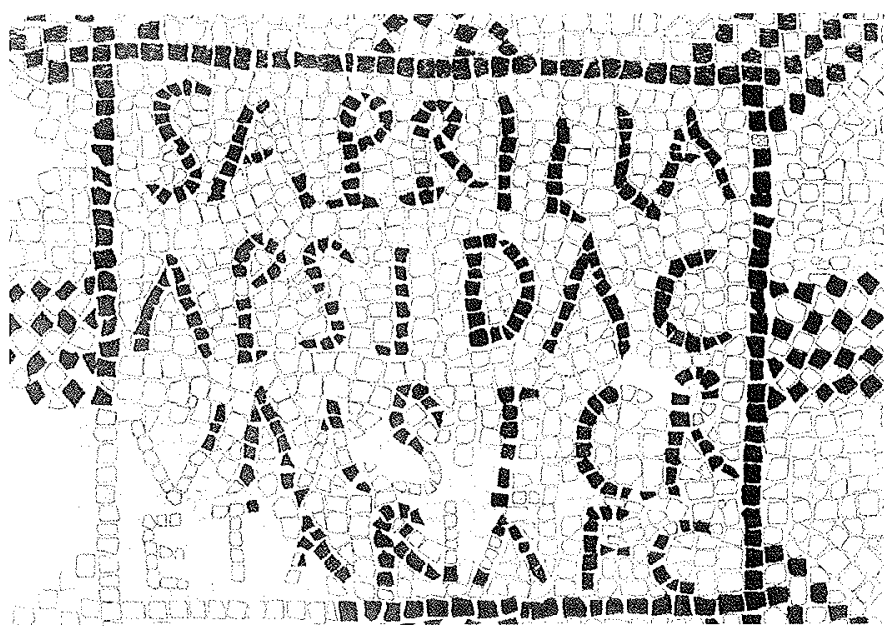

Fig. 3. Iscrizione al centro del campo musivo - quadratini grigi sono posti in corrispondenza delle tessere mancanti

edilizia, è stata aperta una porta nella navata laterale della chiesa) e il retro successivamente edificato, ha la pavimentazione leggermente rialzata in relazione al parterre della chiesa. In questo vano, sotto uno strato di bruciato e di tegole crollate spesso $30 \mathrm{~cm}$., è stato rinvenuto un pavimento in mosaico, in condizioni relativamente buone, dalle dimensioni di $330 \times 330 \mathrm{~cm}$, a tessere bianche e grigio-scure. $\mathrm{Al}$ centro di un semplice campo rettangolare si staglia uno specchio epigrafico di $43 \times 38 \mathrm{~cm}$. con iscrizione su quattro righe.
L'iscrizione è parzialmente danneggiata a causa dell'incendio e del crollo del tetto, ma ciò non ha creato difficoltà nella decifrazione:

\section{SAPRILLA / APSIDAC / VMSECR / ETARIAF(E)C(IT) "Saprilla apsida cvm secretaria fecit"}

Secondo l'iscrizione, una cristiana di nome Saprilla ${ }^{5}$ nome non attestato nel registro paleocristiano dei nomi dell'Italia settentrionale e della Dalmazia, mentre in Istria è presente il cognome "Aprillis") fece edificare, adiacente alla chiesa, il nuovo corpo con l'apside (per noi il termine "apside" significa il vano aggiunto ed il sacrario insieme). Oltre a ciò fu lei ad allestire gli ambienti sussidiari del secretario. Per la prima volta registriamo in situ il termine ad indicare le parti della chiesa paleocristiana secondo la terminologia usata nelle nostre regioni nei secoli V e VI.

$\mathrm{Nel}$ corso dei lavori non abbiamo rinvenuto resti delle suppellettili sacre. Un modesto frammento di pluteo è stato ritrovato su un vicino terreno. L'interno dell'apside è stato da tempo oggetto di scavo e non vi sono tracce di pavimentazione, ma ci è ben noto il suo livello in relazione alla pavimentazione della navata. Neanche all'interno della superficie esplorata del parterre la pavimentazione era stata conservata, ma vi era rimasta traccia di malta tenera, scavata ripetutamente e amalgamata con frammenti musivi bianchi, neri e rossi. Nella fase di ristutturazione del vecchio edificio è stato costruito il basamento di un muro continuo che separava la navata laterale dalla navata centrale della chiesa. Questo basamento continuato reggeva anche un colonnato.

All'interno del parterre della chiesa di Sv. Lucija, B. Fučić ha ritrovato una trentina di frammenti di suppellettili lapidee

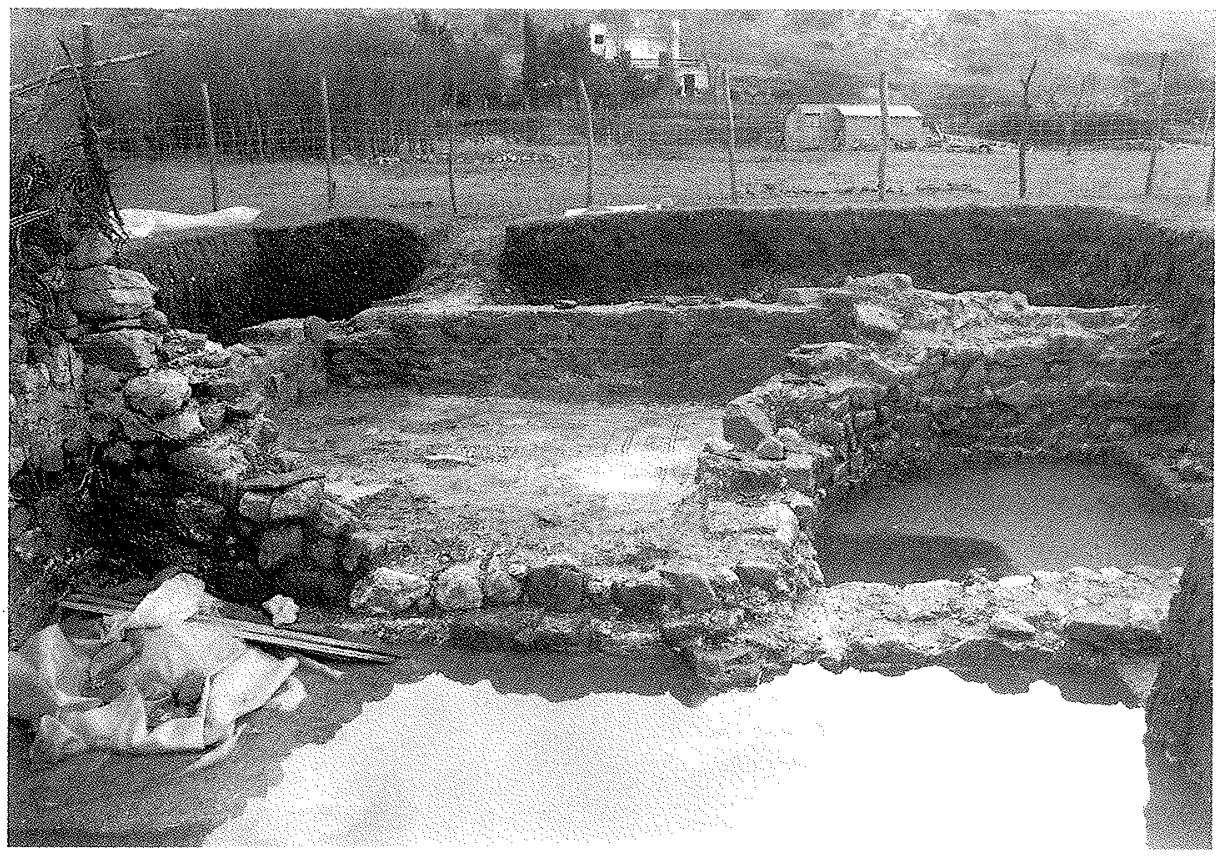

Fig. 4. Chiesa di Su. Nikola, apside 


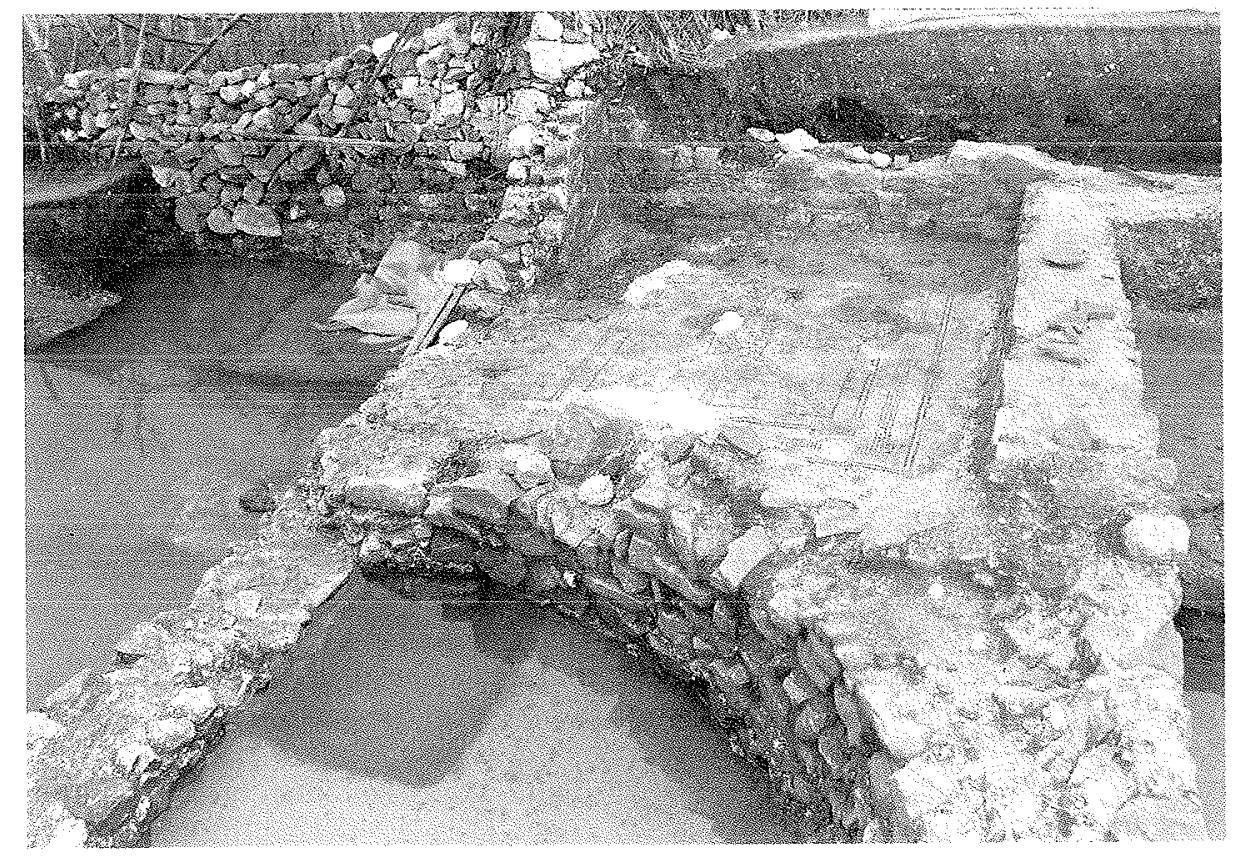

Fig. 5. Chiesa di Sv. Nikola, apside e campo musivo

di origine paleocristiana ${ }^{6}$. I frammenti appartengono a diverse parti di colonnine, capitelli e tavole divisorie d'altare, lavorate in tre tipi di pietra (calcare locale bianco, calcare tenero dell'Istria meridionale e marmo di importazione). Sebbene alcuni pezzi possano appartenere alle suppellettili della modesta cappella votiva paleocristiana eretta nell'ambito del complesso rurale tardoantico, ristrutturata nel IX sec. per le esigenze dell'abbazia dei glagoliti, completamente distrutta durante l'edificazione della chiesa romanica dell'abate Dobrovit, le opere scalpellate in pietra appartengono prevalentemente ad un edificio di culto paleocristiano molto più ampio. Sappiamo dell'esistenza di un vasto complesso paleocristiano con pavimentazioni a mosaico sottostante la cappella romanica di Sv. Marko al centro di Baška. Trattandosi di una regione pietrosa, è tuttavia poco probabile che nel corso del tardo medioevo vi si potesse portare della pietra dalla località di Sv. Marko che dista tre chilometri da Jurandvor. E invece è molto più probabile che almeno una parte del materiale edilizio vi provenisse dall'edificio di Sv. Nikola, ad un chilometro di distanza. Grazie al sondaggio effettuato fino ad ora, è possibile constatare che ai confini della regione di "Mire" vi sono resti di un'ampia basilica paleocristiana a tre navate, con la parte posteriore piatta e con l'abside del sacrario inscritta. Sino a quando non si sarà esaminato l'intero complesso, è prematuro parlare della funzione dell'originario edificio rettangolare. La chiesa ultimata era lunga $28 \mathrm{~m}$.; la larghezza interna della navate laterali risultava di $3 \mathrm{~m}$., la larghezza della navata centrale come quella del sacrario era di 5,20 m mentre la larghezza complessiva (incluso lo spessore dei muri) ammontava a $13,90 \mathrm{~m}$. Le parti di muri attualmente visibili rivelano che l'edificio è dotato di una parete frontale abbastanza inclinata in relazione alla parte posteriore, ma questa deformazione della pianta è una regola dell' architettura paleocristiana. Lungo la parete laterale nordoccidentale della chiesa (con porta secondaria) vi erano degli ambienti sussidiari adiacenti ed una piccola cella votiva.

In base a quanto esposto in questa relazione preliminare, possiamo datare la struttura finale dell'edificio sacro al $\mathrm{V} \mathrm{sec}$. Questo tipo di basilica con l'abside semicircolare inscritta (in passato erroneamente chiamato "tipo architettonico siriano") ${ }^{8}$ è diffuso specie nelle regioni prealpine e settentrionali dell'Italia (soprattutto intorno a Milano ed Aquileia), ma anche nelle vicine zone dell'isola di $\mathrm{Pag}^{9}$ ed in Istria. Nella Croazia settentrionale $^{10}$ e in Dalmazia ${ }^{11}$ questo tipo di basilica appare molto più raramente. Sul finire del IV sec. e agli inizi del V sec., sotto l'incalzante invasione degli Unni e presto anche a causa della migrazione dei Visigoti, le genti dell'entroterra dalmata e della Pannonia occidentale si spostano in massa verso l'Istria e le isole dell'Adriatico. Se accettiamo la tesi che a quell'epoca nella provincia della Dalmazia risultava ormai ultimato il processo di cristianizzazione, con l'afflusso delle nuove genti doveva essersi intensificato il processo di edificazione delle chiese e di organizzazione delle comunità cristiane, in cui un ruolo significativo ricoprivano evidentemente le donne. Per quanto all'epoca del basso impero Baška fosse una piccola comunità di carattere peregrino, del cui nome è tuttora testimonianza l'appellativo della roccaforte sull'altura di "Bag" (nell'antichità probabilmente Vegium), in relazione al grande numero di abitazioni rurali disseminate nel territorio, la località fu abbastanza capace di edificare e conservare ampi edifici paleocristiani. Con i nuovi arrivati dal retroterra continentale 
nel corso dei secoli V e VI, le preesistenti comunità cristiane locali assunsero nuovi stimoli.

Sebbene di respiro ridotto, le ricerche relative alla chiesa di Sv. Nikola nella regione di "Mire" hanno offerto dati significativi per l'archeologia paleocristiana, cosa che dovrebbe senz'altro costituire un ulteriore incentivo per approfondire gli interessi e allargare le ricerche a tutto il patrimonio tardoantico di questa parte dell'isola di Krk, dove fino ad ora siamo a conoscenza (includendo la prima fase edilizia della chiesa di Sv. Nikola nella baia di Ogrul, una chiesa sul castrum e una basilica sottostante l'abitato di Korintija dell'esistenza di sei chiese paleocristiane in tutto.

Traduzione a cura di Suzana Glavaš

\footnotetext{
${ }^{1}$ A. FABER, Osvrt na neka utvrdenja otoka Krka od vremena prethistorije do srednjeg vijeka, in "Prilozi", nn. 3-4, pp. 124-125.

23OLONIĆ-ŽIC, Otok Krk kroz vijekove, Zagreb 1977., p. 391, pp. 393-395.

${ }^{3}$ V. DAUTOVA-RUŠEVLJAN, Antička grobnica u Baški na otoku Krku, in “Arheološki pregled", n. 12, Beograd 1970., pp. 142-143.

${ }^{4}$ Ai lavori hanno preso parte: Ranko Starac (capo gruppo), Ivana Ožanić (laureanda in archeologia), Danko Grigić (documentarista), Radovan Oštrić (sanazione mosaici), Dani Juranić, Anton Mihalić-Komburger e Mario Hrabrić (operai).

${ }^{5}$ J. P. CAILLET, L'évergetisme monumental chrétien en Italie et a ses marges. D'après l'épigraphie des pavements de mosaïque (IV-VII s.), Ecole française de Rome 1993.

"B. FUČ́Ć, Izvještaj o radovima u Jurandvoru kraj Baške na otoku Krku godine 1956. i 1957., Annali JAZU 64, Zagreb 1960., pp. 168-200.

${ }^{7}$ G. C. MENIS, La basilica paleocristiana nella regione delle Alpi orientali, in "Antichità Altoadriatiche" (AAAd) IX, Udine 1976., pp. 375-400.

${ }^{8}$ A. ŠONJE, Bizant i crkveno graditeljstwo u Istri, Rijeka 1981., p. 15-fig. 10, pp. 23-26.

"F. BUŠKARIOL, Prilozi arheološkoj topografiji otoka Paga, ed. HAD, Krk 1985., Zagreb 1989., fig. 2, p. 146.

${ }^{10}$ Rano kršćanstvo u kontinentalnoj Hrvatskoj, in "Od nepobjedivog sunca do sunca pravde", Catalogo della mostra, Arheološki muzej Zagreb/Museo Archeologico di Zagabria 1994., p. 51, fig. 9.

${ }^{11}$ B. MIGOTTI, Prilog datiranju ranokršćanske arhitekture šireg salonitanskog područja, Arheološki vestnik 43, Ljubljana $1992 .$, p. $112,114$.
}

\section{O OTKRIĆU JOŠ JEDNE RANOKRŠĆANSKE CRKVE NA OTOKU KRKU}

SAŽETAK

Tijekom jeseni 1995. obavljeno je pokusno sondiranje lokaliteta "Sv. Nikola" kod predjela Mire, smještenog podno ceste Jurandvor-Baška. Iskopom je istražena polovina začelnog dijela pravokutne građevine, za koju je ubrzo utvrđeno da pripada antičkom objektu adaptiranom i dograđenom za potrebe ranokršćanske zajednice.

Crkva je prostrana longitudinalna trobrodna bazilika ravnog začelnog zida sa upisanom polukružnom apsidom. Unutar jedne od dviju pomoćnih prostorija uz bok apside otkriven je dobro očuvan mozaikom dekorirani pod. Mozaik je načinjen od crno-bijelih tesera koje tvore jednostavan geometrijski motiv unutar kojeg je postavljeno natpisno polje. Natpis na latinskom jeziku govori o kršćanki Saprili koja je dala produžiti gradevinu, tj. načiniti svetište sa pomoćnim prostorijama:

(Saprilla apsida cum secretaria fecit).

Prema do sada prikupljenim elementima možemo pretpostaviti da je crkvena gradevina dovršena tijekom petog stoljeća. 